\title{
Black fungus: a new threat
}

\section{Uddin KN}

Fungal infections, also known as mycoses, are traditionally divided into superficial, subcutaneous and systemic mycoses.

\section{What are systemic mycoses?}

Systemic mycoses are fungal infections affecting internal organs. In the right circumstances, the fungi enter the body via the lungs, through the gut, paranasal sinuses or skin. The fungi can then spread via the bloodstream to multiple organs, often causing multiple organs to fail and eventually, result in the death of the patient.

\section{What causes systemic mycoses?}

Patients who are immunocompromised are predisposed to systemic mycoses but systemic mycosis can develop in otherwise healthy patients. Systemic mycoses can be split between two main varieties, endemic respiratory infections and opportunistic infections.

\section{Endemic respiratory infections}

Fungi that can cause systemic infection in people with normal immune function as well as those who are immunecompromised, include: Histoplasma capsulatum (causes histoplasmosis), Coccidioides immitis (causes coccidioidomycosis), Blastomyces dermatitidis (causes blastomycosis), Paracoccidioides brasiliensis (causes paracoccidioidomycosis), Talaromyces marneffei (causes talaromycosis). These fungi are found in soil and wood debris. Histoplasma is prevalent in Southern USA, Central America, South America, Africa and Asia. Coccidioides and Paracoccidioides are prevalent in Southern USA and Central America. Blastomyces is most often found in North America. Talaromyces marneffei (formerly known as Penicillium marneffei) is endemic in tropical Southeast Asia.

\section{Opportunistic infections}

Fungi that only result in systemic infection in immunocompromised or sick people include:
Candida spp. including non-albicans Candida (causing candidiasis), p. Aspergillus spp. (causing aspergillosis), Cryptococcus (causing cryptococcosis), Mucormycosis previously called zygomycosis caused by Zygomycetes. These fungi are found in or on normal skin, decaying vegetable matter and bird droppings respectively but not exclusively. They are present throughout the world.

\section{Who are at risk of systemic mycoses?}

Immunocompromised people are at risk of systemic mycoses. Immunodeficiency can result from: human immunodeficiency virus (HIV) infection, systemic malignancy (cancer), neutropenia, organ transplant recipients including haematological stem cell transplant, after a major surgical operation, poorly controlled diabetes mellitus, adult-onset immunodeficiency syndrome, very old or very young.

What are the clinical features of systemic mycoses? The clinical features of a systemic mycosis depend on the specific infection and which organs have been affected. General symptoms of illness may include fever, loss of appetite and weight loss.

\section{How are they diagnosed?}

Strong clinical suspicion is vital. The most reliable tests to confirm infection are identification of the organism from representative sterile specimen; skin biopsy for histological (microscopic) analysis and fungal culture. Blood can also be cultured.

\section{What treatment is available for systemic mycoses?}

Once a diagnosis of systemic mycoses has been established, the predisposing state should be reversed, if possible; for example by discontinuing immunosuppressing medications or at least reducing their doses.

Most suitable systemic antifungal medications: amphotericin B, others are fluconazole itraconazole, 
voriconazole, caspofungin. The prognosis depends on the patient's immune function and the extent of infection when treatment has been started.

\section{Black fungus: what is it?}

It is a systemic fungus, called Mucormycetes causing a disease Mucormycosis. Named "black" possibly because the affected area become gray or black. Mucormycosis is an opportunistic infection which represents the third most common angioinvasive fungal infection and is considered as one of the most important medical complications in immunocompromised patients. Mucoraceae are moulds in the environment that transform into hyphal forms in tissues and invade blood vessels producing tissue infarction, necrosis and thrombosis.

\section{Whom to suffer?}

Patients with diabetes, cancer and transplant recipients are the sufferer, because of suppressed immunity. It is not a new disease. In recent days, there is an outbreak, specially, in India with casualties. This is possibly due to immune suppression incurred by COVID-19 or its different modalities of treatments. COVID-19, specially, severe COVID-19 is more prevalent among peoples having diabetes, cancer etc.

\section{How does black fungus spread?}

It prevails everywhere in environment, so habitat is cosmopolitan. Fungal spore grows in soil, rotten leaves, cow dung, rotten food and may stay in air in dry season. The spore enters the body through nose, mouth and lungs and the breach of skin. It invades basically those are immunosuppressed.

\section{Which part of body mostly affected?}

Nose, sinuses, orbits, brain (rhinoorbitocarebral infection) are mostly affected; respiratory tract, lungs, gastrointestinal tract and skin may also be affected. Rhinocerebral and pulmonary

involvements are the two most common forms. Though mucormycosis has been diagnosed since many years, recently large numbers of cases are being identified in patients recovered from COVID-19. Patients with COVID-19 present with high levels of inflammatory cytokines and associated with impaired cell-mediated immune response, which lead to increased susceptibility to fungal co-infections. Also, the use of corticosteroids and other immunosuppressive agents and oxygen delivery devices have posed mucormycosis as an emerging threat for patients with COVID-19. Casualty is very high, may exceed $50 \%$. Mortality rate due to gastrointestinal mucormycosisis as high as $85 \%$.

Mucormycosis and other opportunistic infections among patients suffering and recovering from

COVID-19 have emerged as a challenging health issue throughout the globe, specially more in

India. A case of post-COVID pulmonary mucormycosis is reported from Bangladesh. Several factors are responsible for such an increased incidence of invasive fungal infections among patients recovering from COVID-19, which include severe pulmonary alveolar damages in COVID-19 that facilitate fungal invasion, immune dysregulations in COVID-19, invasive mechanical ventilations may facilitate entry of opportunistic pathogens, so does immunosuppressive drugs used in COVID-19, prolonged hospital stay and injudicious antibiotic prescription.

\section{How do you diagnose mucormycosis?}

1) clinical features 2) CT scan and/or MRI, depending on organ involvement 3) swab from lesion or tissue biopsy specimen and culture; cultures may be negative in 40-50 percent of clinical cases. Likewise, as Mucorales are common contaminants of airways, isolation of these organisms from sputum must be interpreted with caution.

\section{Treatment}

Antifungal drugs: amphotericin B is the drug of choice and very much effective but it is highly expensive. Posaconazole or isavucanazole may be used as alternative (fluconazole, itraconazole or voriconazole not effective)

Surgery may be life-saving in some cases.

Follow-up curettage may be essential for some cases.
Khwaja Nazim Uddin
Professor
Department of Internal Medicine
BIRDEM General Hospital, Dhaka, Bangladesh
Email:knuddin61@gmail.com 